\title{
Methods for evaluation of mechanical stress condition of materials
}

\author{
Yordan Mirchev ${ }^{1, *}$, Pavel Chukachev ${ }^{2}$, and Mitko Mihovski ${ }^{1}$ \\ ${ }^{1}$ Institute of Mechanics of Bulgarian Academy of Science, 1113 Sofia, Bulgaria \\ ${ }^{2}$ Multitest Ltd., 9000 Varna, Bulgaria
}

\begin{abstract}
Primary attention is given to the following methods: method by drilling cylindrical holes (drill method) and integrated ultrasonic method using volume (longitudinal and transverse), surface, and sub-surface waves. Drill method allows determination of residual mechanical stress in small depth of material surfaces, assessing type, size, and orientation of principal stresses. For the first time, parallel studies are carried out of mechanical stress in materials using the electroacoustic effect of volume, surface and sub-surface waves on the one hand, and effective mechanical stresses on the other. The experimental results present electroacoustic coefficients for different types of waves in the material of gas pipeline tube of $243 \mathrm{~mm}$ diameter and $14 \mathrm{~mm}$ thickness. These are used to evaluate mechanical stresses in pipelines, according to active GOST standards.
\end{abstract}

\section{Introduction}

In recent years, scientific researches and their practical use has contributed to create a significant number of effective methods for assessing mechanical stresses based on dependencies between residual and effective stresses in material and certain physical characteristics (coercive force, magnetic memory, magnetic noise, propagation velocity of ultrasound waves, X-ray diffraction, etc.). There are also a number of semi-destructive methods, among which the so-called "drill method", is also successfully applied $[7,8]$.

In this study, complex investigations were carried out using volumetric, surface and subsurface ultrasonic waves for the evaluation of mechanical stress condition (MSC) in pipeline, and examinations by the semi-destructive "drill method". The main task in studying the MSC of pipelines is to determine axial $\sigma_{z}$ and tangential (along the circumference) $\sigma_{\mathrm{h}}$ mechanical stresses.

The aim is to propose an algorithm to estimate the relative variation of MSC in surface and sub-surface layers of pipeline material, as well as the relative variation of MSC in material for the whole pipeline wall thickness.

* Corresponding author: mirchev@imbm.bas.bg 


\section{Method to investigated mechanical stress condition}

\section{1 "Drill method"}

The hole drilling method is one of the widely used semi-destructive methods for determining the residual stresses in the surface layer of material. Damage caused to the material is a not deep small diameter cylindrical hole that is permissible or can be repaired $[7,8]$.

The method is performed by drilling a hole of up to $2 \mathrm{~mm}$ depth using cylindrical drill (of up to $1.8 \mathrm{~mm}$ diameter) in the investigated area of material. By means of properly attached strain gauges around the hole, induced deformations are determined at each hole drilling step. From deformations obtained, residual stresses are estimated using proper algorithms. Calibration constants are calculated for each strain gauge rosette used. Good knowledge of the modulus of elasticity and mechanical characteristics of the material is involved.

The method is mainly applied to isotropic materials. The recommended depth of the cylindrical hole is up to 0.5 times $\mathrm{D}$, where $\mathrm{D}$ is the diameter of the drill used [7].

\subsection{Ultrasonic method}

Acoustic strain gauge measurements present the methods for determining the stresseddeformed state of the material by investigating the characteristics of the elastic waves propagating in material (amplitude, velocity, polarization, etc.). These dependencies are subject to study in non-linear acoustics and are related to the existence of the following phenomena:

- Nonlinear interaction of the elastic waves associated with the transmission of energy by interacting waves in the heterodyne frequency wave.

- Refraction of the sound wave in the presence of mechanical stresses where the vector describing the energy flow does not coincide with the wave propagation vector.

- Modulation of the sound wave in wave propagation in media of periodically varying parameters, resulting in wave phase, amplitude and frequency variations.

- Acoustic elasticity related to the dependence of elastic waves distribution velocity in solids on mechanical stresses (strains), type of wave polarization and the mutual orientation of the wave vector relative to the direction of mechanical effects.

A prerequisite for the application of acoustic-elastic effect methods is the variation in the elastic properties of the material and the propagation velocities of the ultrasound waves in a material in the absence or presence of mechanical stresses [1-6].

\section{Technical implementation of the methods}

\section{1 "Drill method"}

According to the operating instructions of the unit MTS 3000 RESTAN [8] made by SINT Technology- Italy it is recommended to work with strain gauge rosettes of type $A, B$ or $C$. During the experiments carried out in this work, a type $A$ rosette is used complying with the requirement for drill depth and recommended depth increments.

In fig. 1a, a schematic diagram is shown of fixing strain gauges in three-element rosette of type $A$ used to drill a hole to evaluate residual mechanical stresses. Here, $\sigma_{\max }, \sigma_{\min }$ are maximum and minimum residual stresses around the drilled hole. 
Fig. 1b shows block-scheme of typical hole drilling device. It presents an optical device for centering the tool holder and drilling tool. There are a number of requirements to the device, including:

- The hole shall be drilled concentric to the circle of strain gauges of diameter $D$ with accuracy \pm 0.004.D.

- Drills or cylindrical hard-alloy mills driven by an electric motor are used with a rotational speed of up to $500000 \mathrm{rpm}$.

- Radial clearance angles of the tool cutting edges ensuring uniformity in depth up to $1 \%$ of tool diameter.

- Drilling of the hole is carried out under constant temperature conditions.

- The measuring instrument for recording the deformations shall have a resolution of \pm $1.10^{-6}$ with the same repeatability of measurements.

After the tests, the residual stresses in the material are calculated, according to the requirements of ASTM E 837-08 [7].

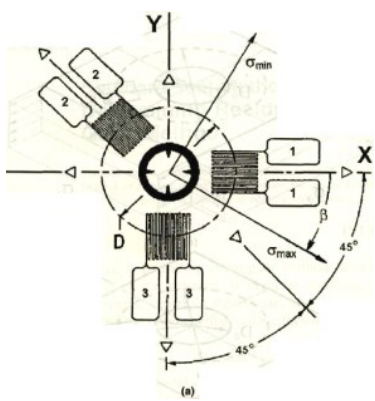

a)

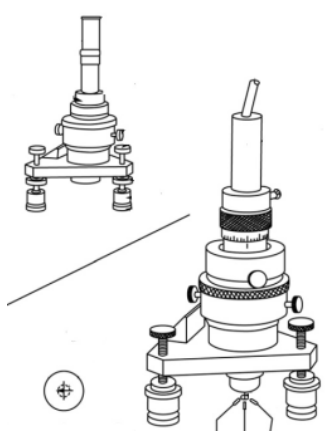

b)

Fig. 1. a) Three grid rosette, type $A$ and b) block-scheme of hole drilling device.

\subsection{Ultrasonic method}

MSC determination is performed by determining the relative variation of velocity or times of ultrasonic wave propagation in the material of the pipelinece. The relative variation is read against material in pipe of MSC assumed as base. The relation between the relative velocity variation and the mechanical stress variations is determined.

In the present work, three approaches to determine MSC are used - in the surface, subsurface, and the entire volume of the pipe material wall. The relative variations of the following are studied times of travel of:

- Transverse waves, polarization along and transverse to generant of the pipe and longitudinal wave propagating along the radius of the pipe.

- Surface Rayleigh wave.

- Sub-surface lateral wave.

Within the first approach, the MSC is determined over the entire wall volume of the pipe for the investigated area in which the waves propagate. The times of travel are determined by the echo method. With surface Rayleigh waves and sub-surface waves, MSC is investigated in surface and sub-surface layer respectively of the pipe wall, the times being determined by through transmission method.

GOST R 52890-2007 [5] regulates the basic requirements for the determination of a mechanical stress condition by volumetric ultrasound waves propagating along the radius of the controlled pipes. The standard is applicable to steel pipes (welded and seamless) where 
ratio of wall thickness to outside diameter is 20 and to pipes of diameter over $325 \mathrm{~mm}$. The standard has been drawn up based on scientific researches in Mechanical Engineering Research Institute of the Russian Academy of Sciences in Nizhniy Novgorod conducted by Nikitina N. E. [1].

The investigations of the times of the ultrasound waves propagated in the material are carried out on pre-prepared reference samples of thickness from $13.817 \mathrm{~mm}$ to $15.545 \mathrm{~mm}$, cut out along the pipe away from the welded joint [9]. The samples are prepared by grinding machine Okamoto from pipe X52N PSL2/L360 and marked by numbers FE01, FE02, FE03 and FE04. Appearance of samples to determine times of wave travel in a material under tensile load is shown in fig. 2.

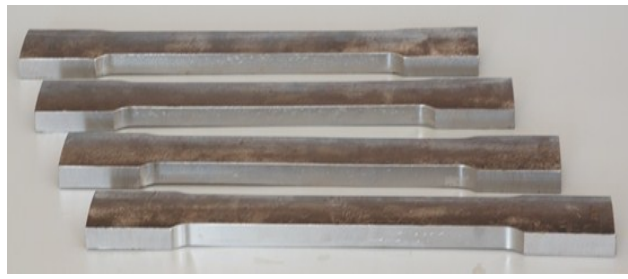

Fig. 2. Reference samples for evaluation of acoustic elastic factors.

Mechanical tests of material are carried out in advance in order to assess the yield point, creep and rupture limits. On this basis, the load and its velocity are regulated (to avoid the influence of the temperature effect when changing the velocity of the wave propagation). Stressing the tensile samples is carried out by EU100 machine. Loading mode is set so that it does not penetrate the metal creep area.

Determination of ultrasonic wave travel time in material is carried out by flaw detector Omniscan MX and probes for transverse waves of type V156-RM, for longitudinal of type V110-RM, for surface - type A574S, for sub-surface - type C567-SM.

Calipers, gauge blocks and clamping devices of high accuracy are used. Coupling the probes to the test samples is one of the most important tasks in order to achieve optimum acoustic contact and reduce the coupling effect on time determination. For each of the probes in use special clamping units are made equipped with magnets of clamping force $4 \mathrm{~kg}$. Probes and clamping units are illustrated in fig.3 (attached to a pipe). In fig.4, probes are placed on a reference sample.

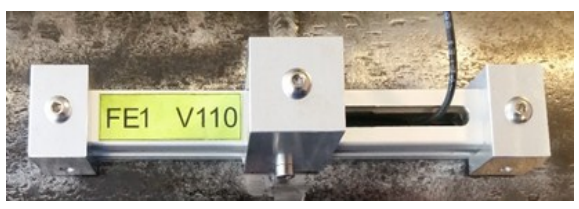

a)

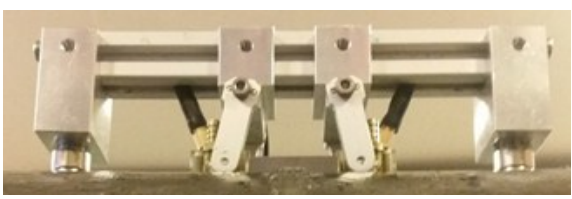

b)

Fig. 3. Clamping units for (a) normal probe and (b) subsurface.

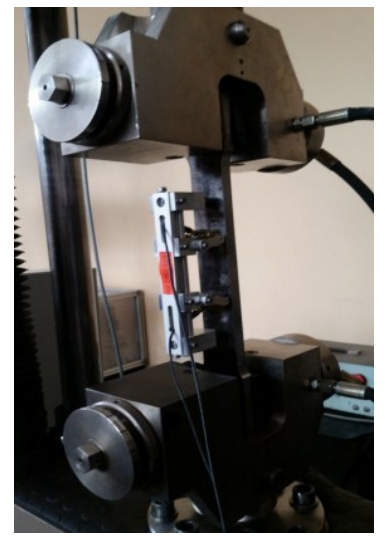

Fig.4. Setup of sensors attached to test sample and sample to the clamps of the test machine. 
In the process of acoustic measurements shown in fig. 4, records of the load-time dependencies are made. With short interruptions of the mechanical tensile loader, using the ultrasonic unit, 3 to 5 independent measurements are made to determine the times of the waves travelling in the material. The number of measurements shall be repeated until the required uncertainty is reached.

On the basis of the acoustic paths data and the travel times for the respective load, the ultrasonic wave propagation velocities are calculated. The dependencies of the relative variation of velocities or travel times of ultrasound waves from load for surface and subsurface waves are plotted. When working with volumetric longitudinal and transverse waves, dependence of the calculated mechanical stresses is plotted using the theoreticallyexperimentally obtained acoustic-elastic factors.

\section{Results of investigations of the reference samples to the ultrasonic method}

The relative variations in the times for ultrasonic wave propagation in the material were determined. The time for the corresponding velocity in unstressed condition of the sample is accepted as a base time. The relative variation in \% of times is calculated by the following equation

$$
\Delta \tau, \%=\frac{\tau(P \neq 0 M P a)-\tau(P=0 M P a)}{\tau(P=0 M P a)} * 100
$$

Relative variations of times calculated from tensile stresses applied of $P=50 M P a \div$ $300 M P a$, by $50 M P a$ steps are presented in fig.5. The calculations are performed for values of the times determined by five independent measurements at a confidence level of $95 \%$. The relative variations of the transverse wave are marked by transverse 0 and transverse 90 with polarization respectively in the direction of the tensile stresses (along the pipe generant of which the reference samples are made) and perpendicular to the direction of the tension stresses.

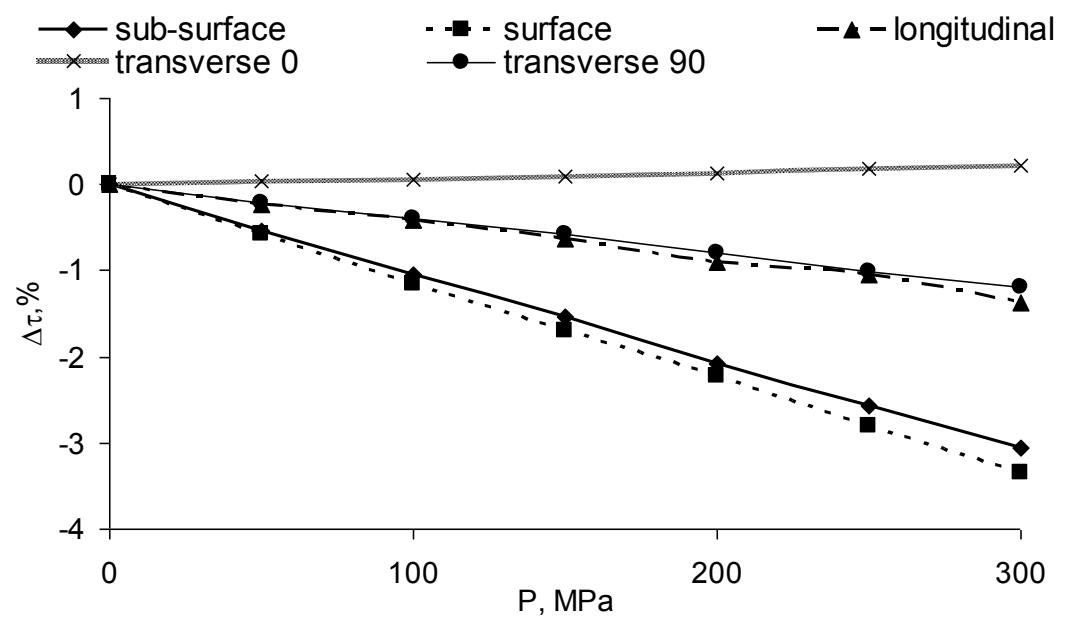

Fig. 5. Relative variation of travel times of ultrasonic waves in material of samples with mechanical tensile loading. 
Dependencies in fig. 5 can be used as comparison curves for determination of the relative variation of mechanical stresses in the investigated area of the object.

In determining the mean variation of mechanical stresses across the wall thickness of the object under investigation, an algorithm is used specified in the standards GOST R 52890-2007 [5] and GOST R 52731-2007 [6]. The use of this algorithm disregards the wall thickness of the tested object in experimental determination of the travel times of longitudinal and two mutually polarized at $90^{\circ}$ transverse waves. Their values are used to calculate acoustic-elastic factors. By the algorithm and the dependencies given in $[1,10]$ acoustic-elastic factors $\kappa_{1}, \kappa_{2}, \kappa_{3}$ are calculated. Factors in uniaxial mechanical stressed condition are calculated by the following equations

$k_{1}=\frac{\frac{L}{\tau_{1}} \cdot \frac{\tau_{01}}{L_{0}}-1}{\sigma_{z}}, \quad k_{2}=\frac{\frac{L}{\tau_{2}} \cdot \frac{\tau_{02}}{L_{0}}-1}{\sigma_{z}}, \quad k_{3}=\frac{\frac{L}{\tau_{3}} \cdot \frac{\tau_{03}}{L_{0}}-1}{\sigma_{z}}$,

where $L_{0}$ and $L$ are respectively, the paths of the propagating waves before and after the action of mechanical stresses, $\tau_{0 i}$ and $\tau_{i}$ - the wave propagation times, respectively, before and after application of the tensile stress $\sigma_{z}$ in longitudinal direction of reference samples. Here $k_{1}$ is related to propagation of transverse waves at $0^{\circ}$ polarization, $k_{2}$ - transverse wave at $90^{\circ}$ polarization and $k_{3}-$ longitudinal wave. The polarization given for transverse wave at $90^{\circ}$ is in direction of the tensile stresses.

Using the experimental data obtained for the propagation times of the longitudinal and transverse polarized waves to equations (2), acoustic-elastic factors $\kappa_{1}, \kappa_{2}, \kappa_{3}$ are calculated. In Table 1, their values are listed as obtained for each load value $\mathrm{P}$.

Table 1. Calculated acoustic-elastic factors $k_{1}, k_{2}$ and $k_{3}$

\begin{tabular}{|c|c|c|c|}
\hline $\begin{array}{c}\text { Load P, } \\
\mathbf{M P a}\end{array}$ & $\begin{array}{c}\mathbf{k}_{\mathbf{1}}, \\
\mathbf{1 0}^{-6} \mathbf{M P a}^{-\mathbf{1}}\end{array}$ & $\begin{array}{c}\mathbf{k}_{\mathbf{2}}, \\
\mathbf{1 0}^{-6} \mathbf{M P a}^{-\mathbf{1}}\end{array}$ & $\begin{array}{c}\mathbf{k}_{\mathbf{3}}, \\
\mathbf{1 0}^{-6} \mathbf{M P a}^{-\mathbf{1}}\end{array}$ \\
\hline 50 & 6.659 & -40.336 & -47.557 \\
\hline 100 & 6.661 & -40.225 & -41.001 \\
\hline 150 & 6.663 & -39.435 & -41.631 \\
\hline 200 & 6.109 & -39.541 & -45.093 \\
\hline 250 & 6.667 & -40.013 & -42.024 \\
\hline 300 & 6.670 & -39.933 & -45.594 \\
\hline
\end{tabular}

According to the values of acoustic-elastic factors $k_{1}, k_{2}$ and $k_{3}$ for each load value $P$ the coefficients $K_{1}$ and $K_{2}$ are calculated to the equations given in [1, 10]

$$
\begin{aligned}
& K_{1}=\frac{k_{1}-k_{3}}{\left(k_{1}-k_{3}\right)^{2}-\left(k_{2}-k_{3}\right)^{2}}, \\
& K_{2}=\frac{k_{2}-k_{3}}{\left(k_{1}-k_{3}\right)^{2}-\left(k_{2}-k_{3}\right)^{2}} .
\end{aligned}
$$

In Table 2, calculated values for $K_{l}$ and $K_{2}$ are listed for each load step. 
Table 2. Calculated values for $K_{1}$ and $K_{2}$

\begin{tabular}{|c|c|c|}
\hline $\begin{array}{c}\text { Load P, } \\
\mathbf{M P a}\end{array}$ & $\begin{array}{c}\mathbf{K}_{\mathbf{1}}, \\
\mathbf{1 0}^{\mathbf{5}} \mathbf{M P a}\end{array}$ & $\begin{array}{c}\mathbf{K}_{\mathbf{2}}, \\
\mathbf{1 0}^{5} \mathbf{M P a}\end{array}$ \\
\hline 50 & 0.1878 & 0.0250 \\
\hline 100 & 0.2099 & 0.0033 \\
\hline 150 & 0.2075 & 0.0094 \\
\hline 200 & 0.1977 & 0.0214 \\
\hline 250 & 0.2057 & 0.0085 \\
\hline 300 & 0.1936 & 0.0210 \\
\hline
\end{tabular}

The following mean values are obtained $K_{1}=0.2004 .10^{5} \mathrm{MPa}$ and $K_{2}=0.0148 .10^{5}$ $M P a$.

\section{Evaluation of residual mechanical stresses in pipe of pipeline}

Distribution of mechanical stresses is studied along the length and circumference in submitted pipe of material X52N PSL2/L360 from pipeline of diameter $D=273 \mathrm{~mm}$ and wall thickness $14 \mathrm{~mm}$. The pipe is not subjected to mechanical stresses. However, it is presumed that along the length and circumference of the pipe there are irregularly spread mechanical stresses.

Particularly significant changes are expected in the part of the pipe located in the near seam zone and in the welded joint itself.

\section{1 "Drill method"}

The measurements are carried out with the MTS 3000 RESTAN system, manufactured by company SINT Technology - Italy. The method is developed based on ASTM E 837-08 [7]. Software product is also used of this company.

Measurements are taken in 4 points. Experiments $E 1$ and $E 2$ are carried out with drilling holes in the inner surface of the pipeline, and $E 3$ and $E 4$ in the outside surface. Summarized obtained results of investigations in the inner and outside surface are listed in table 3 and table 4. Safety factor $n=\sigma_{s} / \sigma_{e}$ is higher for measurements in inner surface and lower - for outer surface. In all cases $n>2$.

Table 3. Results of investigations of mechanical stresses in the inner surface of the pipe.

\begin{tabular}{|c|c|c|c|c|c|c|}
\hline Experiment & $\begin{array}{c}\boldsymbol{\sigma}_{\min }=\boldsymbol{\sigma}_{\mathbf{3}}, \\
\mathbf{N} / \mathbf{m m}^{2}\end{array}$ & $\begin{array}{c}\boldsymbol{\sigma}_{\max }=\boldsymbol{\sigma}_{1}, \\
\mathbf{N} / \mathbf{m m}^{2}\end{array}$ & $\begin{array}{c}\boldsymbol{\sigma}_{2}, \\
\mathbf{N} / \mathbf{m m}^{2}\end{array}$ & $\boldsymbol{\beta}, \mathbf{d e g}$ & $\begin{array}{c}\boldsymbol{\sigma}_{\mathrm{e}}=\boldsymbol{\sigma}_{\mathbf{1}}-\boldsymbol{\sigma}_{3}, \\
\mathbf{N} / \mathbf{m m}^{2}\end{array}$ & $\mathbf{n}$ \\
\hline$E 1$ & -84.4 & 20.89 & 0 & 31.15 & 105.29 & 3.42 \\
\hline$E 2$ & 69.29 & 121.50 & 0 & 79.75 & 121.5 & 2.96 \\
\hline
\end{tabular}


Table 4. Results of investigations of mechanical stresses in the outer surface of the pipe.

\begin{tabular}{|c|c|c|c|c|c|c|}
\hline Experiment & $\begin{array}{c}\boldsymbol{\sigma}_{\min }=\boldsymbol{\sigma}_{\mathbf{2}}, \\
\mathbf{N} / \mathbf{m m}^{2}\end{array}$ & $\begin{array}{c}\boldsymbol{\sigma}_{\max }=\boldsymbol{\sigma}_{\mathbf{1}}, \\
\mathbf{N} / \mathbf{m m}^{2}\end{array}$ & $\begin{array}{c}\boldsymbol{\sigma}_{3}, \\
\mathbf{N} / \mathbf{m m}^{2}\end{array}$ & $\boldsymbol{\beta}, \mathbf{d e g}$ & $\begin{array}{c}\boldsymbol{\sigma}_{\mathrm{e}}=\boldsymbol{\sigma}_{\mathbf{1}}-\boldsymbol{\sigma}_{\mathbf{3}}, \\
\mathbf{N} / \mathbf{m m}^{2}\end{array}$ & $\mathbf{n}$ \\
\hline$E 3$ & 46.8 & 166.4 & 0 & -24.49 & 166.44 & 2.16 \\
\hline$E 4$ & 72.85 & 159.3 & 0 & -12.46 & 159.3 & 2.26 \\
\hline
\end{tabular}

The data analysis shows that $\sigma_{\min }, \sigma_{\max }$ and $\sigma_{e}$ are larger on the outer surface of the material. The residual stresses in material of pipeline are relatively high due to the method used to drill the pipeline and the presence of heterogeneities, non-metallic inclusions and different structure in different areas of pipeline.

\subsection{Ultrasonic method}

Measurements of initial data parameters (velocities of propagation of longitudinal, transverse, surface and subsurface waves) are carried out along the length of three generants of the pipe, located at $0^{\circ}, 90^{\circ}$ and $180^{\circ}$ to circumference. The zero reading along the generant coincides with central part of the welded joint. The weld reinforcement was removed by mechanical cleaning as they do in advance for many responsible welded joints, for example in Nuclear Power Plants.

Along the generant, measurements are presented from $0 \mathrm{~mm}$ (in the center of the welded joint) to $350 \mathrm{~mm}$ by $50 \mathrm{~mm}$ steps. In some cases, additional measurements have also been carried out and immediately in the welded joint with a step of $5 \mathrm{~mm}$.

Fig.6a presents the obtained dependencies for changes in the propagation time of the surface Rayleigh waves $\tau_{R}$ to length $L$ of the pipe. The obtained values are designated by $0^{\circ}$, $90^{\circ}$ and $180^{\circ}$ with directions $0^{\circ}, 90^{\circ}$ and $180^{\circ}$ to circumference of the pipe. The spacing between fronts of the probes C567SM used is $10 \mathrm{~mm}$.

Fig.6b presents dependence $\tau_{R}$ on location of the middle of the distance between fronts of the probes to generant placed at $90^{\circ}$ to circumference of the pipe. Measurements were made at steps of $5 \mathrm{~mm}$ from the center of the welded joint to $L=50 \mathrm{~mm}$ to generant of the pipe.

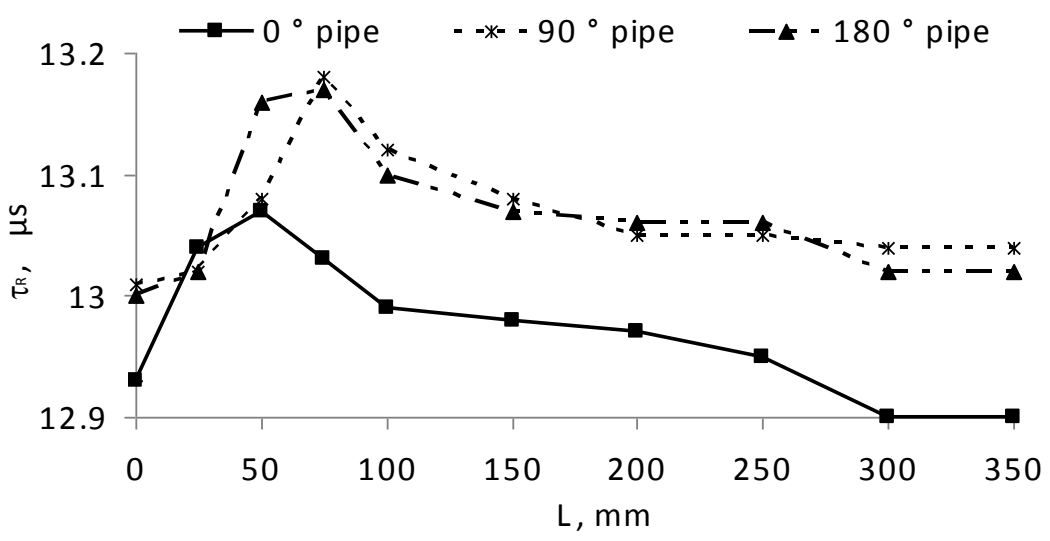

a) 


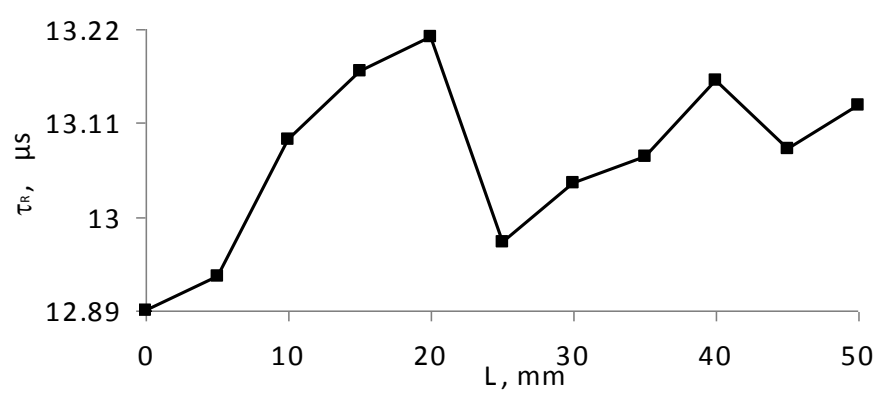

b)

Fig. 6. Dependencies of the travel time of surface wave $\tau_{R}=F(L)$

Significant differences in travel times are observed for the area of the welded joint and the surrounding area.

Fig.7a presents the dependence of the travel time $\tau_{\Pi}$ of the subsurface wave measured along the generant $L$. Spacing between the probes for subsurface waves is $30 \mathrm{~mm}$.

Fig. 7b, Fig.7c and Fig.7d present the dependencies for the travel times, respectively of transverse $\tau_{t}\left(0^{\circ}\right)$, (direction of polarization to generant), transverse $\tau_{t}\left(90^{\circ}\right)$, (direction of polarization transverse to generant) and longitudinal $\tau_{l}$ waves, for various points on the generant $L$, located at $0^{\circ}, 90^{\circ}$ and $180^{\circ}$ on the circumference of the pipe. It is worth noting that there is a difference in the thickness of the pipe at different points in which the times of distributed waves are determined.

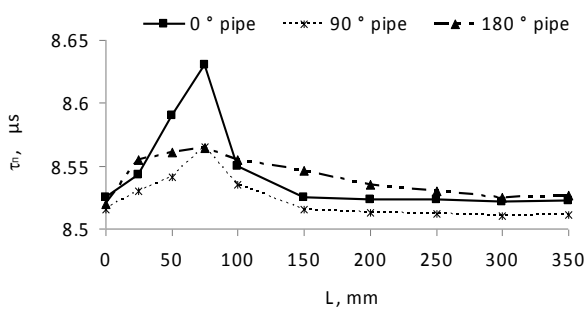

a)

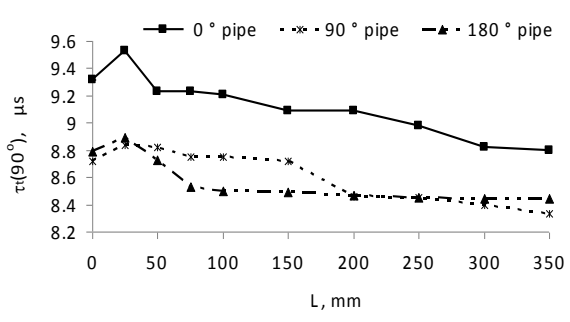

c)

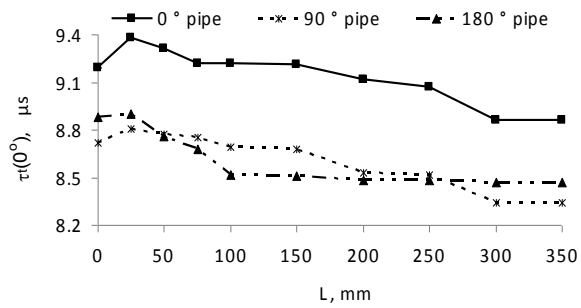

b)

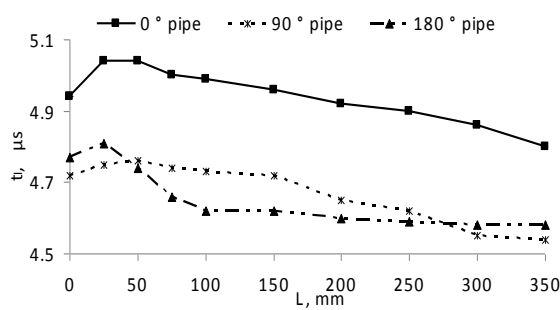

d)

Fig.7. Dependencies $\tau_{\Pi}=F(L)$ a), $\tau_{t}\left(0^{\circ}\right)=F(L)$ b), $\tau_{t}\left(90^{\circ}\right)=F(L)$ c) and $\left.\tau_{l}=F(L) \mathrm{d}\right)$

Using the dependencies for the times of surface and subsurface waves in material of reference samples and tested pipe distribution of mechanical stressed condition is determined in the points investigated of the surface and subsurface layer of the pipe. 
Based on values obtained of times of travel of longitudinal $\tau_{3}$ and transverse $\tau_{1}$ (in direction of polarization of wave to generant) and $\tau_{2}$ (in direction of polarization of wave transverse to generant) waves in reference samples distribution of residual equivalent stresses is calculated (to generant $\sigma_{\mathrm{z}}$ and transverse to generant along the circumference of the pipe $\sigma_{\mathrm{h}}$ ) for a pipeline. In this case mechanical stresses are equivalent and related to the whole thickness of pipeline wall. Applying the mean values of theoretically-experimentally obtained acoustic-elastic factors $K_{l}$ and $K_{2}$ the relative variations of mechanical stresses are expressed by the following equations

$$
\begin{gathered}
\sigma_{z}=K_{1} \cdot \Delta_{1}-K_{2} \cdot \Delta_{2}, \\
\sigma_{h}=K_{1} \cdot \Delta_{2}-K_{2} \cdot \Delta_{1},
\end{gathered}
$$

where

$$
\begin{aligned}
& \Delta_{1}=\left(\frac{\tau_{3} \cdot \tau_{01}}{\tau_{1} \cdot \tau_{03}}-1\right), \\
& \Delta_{2}=\left(\frac{\tau_{3} \cdot \tau_{02}}{\tau_{2} \cdot \tau_{03}}-1\right),
\end{aligned}
$$

$\tau_{1}, \tau_{2}$ and $\tau_{3}$ are, respectively, times of travel of both transverse and longitudinal waves, $\tau_{01}$, $\tau_{02}$ and $\tau_{03}$ - times of travel in absence of mechanical stresses.

The distribution of residual stresses in the pipe investigated is presented in fig. 8 . The stress distributions are observed along the generant L from the weld center. Generant is located at $90^{\circ}$ to circumference of the pipe. The values of residual mechanical stresses are compared with that located in the weld center. They are determined using ultrasonic methods and theoretically-experimentally obtained acoustic-elastic coefficients for the reference samples in the present work.

Fig. 8a gives the values of the residual stresses $\sigma_{z}$, acting in direction of the generant of pipeline. The residual stresses obtained from determination of times of travel in material of surface, subsurface and volumetric ultrasonic waves are designated respectively, by surface, subsurface and volumetric.

Fig.8b shows the values of the residual stresses $\sigma_{\mathrm{h}}$, acting along the circumference of the pipe (perpendicular to generant). Here, the residual stresses are determined from the obtained times of travel of volumetric ultrasonic waves in material and acoustic-elastic coefficients obtained from the reference samples by equation (6). 


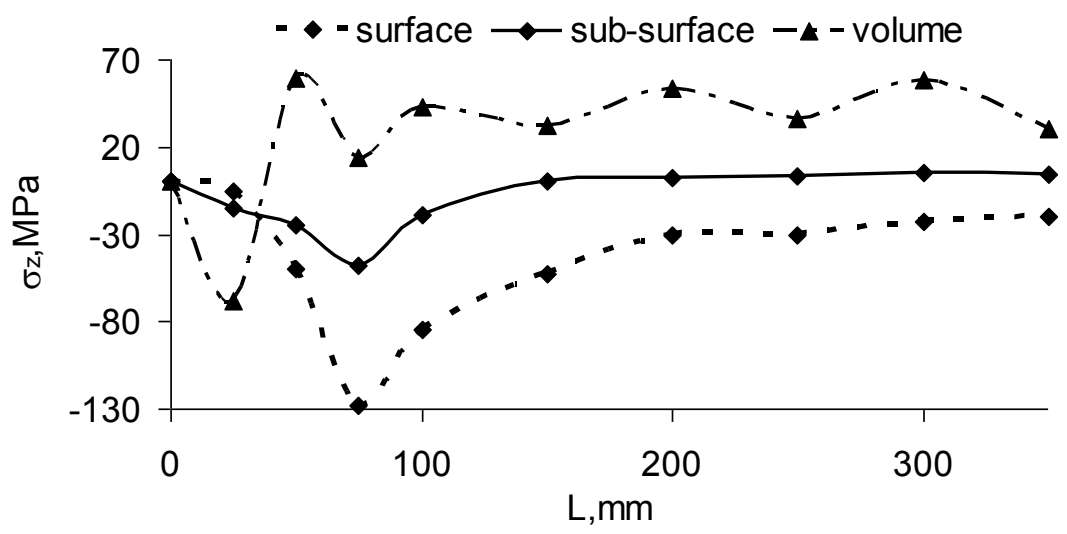

a)

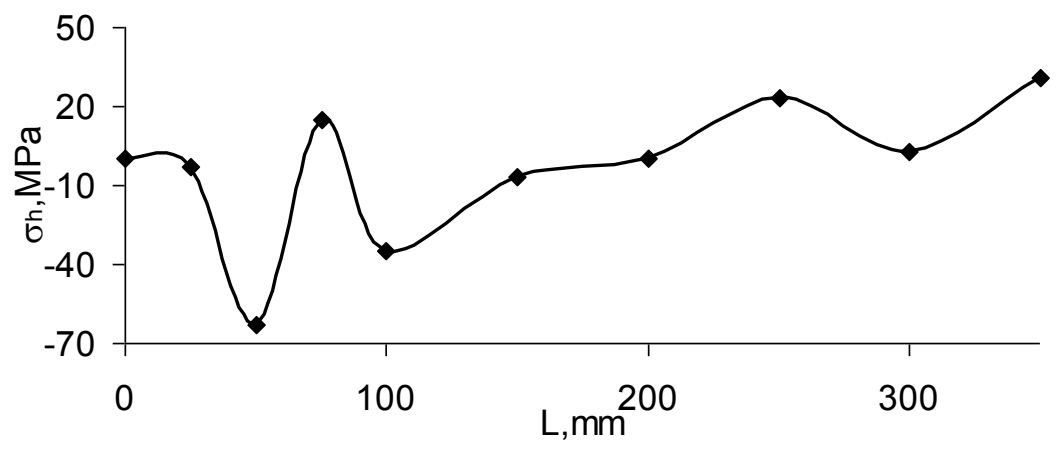

b)

Fig.8. Distribution of the residual stresses to generant L, a) acting in direction of generant and $b$ ) along the circumference of the pipe (perpendicular to generant).

The most significant are the variations of the residual stresses $\sigma_{z}$ in surface layer by up to $128 \mathrm{MPa}$ and of equivalent residual stresses for the whole volume in radial direction in depth by up to $140 \mathrm{MPa}$ (Fig.8a). In subsurface layer the residual stresses obtained vary by up to $50 \mathrm{MPa}$.

The dependence on the distribution of the equivalent volumetric stresses in fig. $8 \mathrm{a}$ is non-monotonic and different from that for the surface and subsurface stresses, which is due to the fact that the residual stresses in wall depth vary non-monotonously from one surface to the other. This fact affects considerably the values of equivalent stresses determined by volumetric ultrasonic waves, especially in the welded joint and weld surrounding area of 75 $m m$ length.

The dependence for the residual stress distribution in fig. $8 \mathrm{~b}$ varies by up to $80 \mathrm{MPa}$.

The complex use of the "drill method" with the acoustic strain gauge measurements allows to obtain true values of the residual stresses from their relative variations in the surface layer of material. This is done by determining the residual stresses using the drill method for one of the points investigated by acoustic strain gauge measurements in the surface for the same direction of action determined by acoustic strain gauge measurements.

Logically, the values obtained for the different measurement points and by different method used differ. The pipeline volume in which the propagation times for the various 
types of waves are obtained is different - the surface layer, the subsurface layer and the volume in depth.

Nevertheless, the obtained values show the applicability of the developed algorithm for the evaluation of the mechanical stresses in investigated material from an object.

\section{Conclusion}

Basic ultrasonic methods, as well as a semi-destructive method of MSC assessment were presented. Acoustic elastic coefficients for the test material and changes in velocities from mechanical stresses for surface and subsurface waves were obtained.

A MSC evaluation algorithm has been proposed together with producing and performing preliminary ultrasonic tests of reference samples to obtain acoustic elastic coefficients and comparative curves of times of travel of surface and subsurface ultrasound waves to determine relative variations of mechanical stresses in the investigated material.

The proposed algorithm is applied to a part of the pipeline for which the relative variations of the residual mechanical stresses along the generant, acting along the generant and the circumference of the pipe are determined

The complex use of "drill method" and ultrasonic strain measurements allows to determine the actual residual stresses in the surface layer of the investigated material.

\section{References}

1. N. E. Nikitina, Acoustic-elastic. Application in practical experiance (N. Novgorod: TALAM 2005)

2. V. M. Bobrenko, A.N. Kutsenko, I.P. Malahov, Acoustical testing of mechanical stress (Odesa: Astroprint 1997)

3. V. M. Bobrenko, M. S. Vangeli, A. N. Kutsenko, Acoustical stress measurement (Kishinev, Shtintsa, 1991)

4. A. N. Guz, F.G. Mahort, O. I. Gushta, V. K. Lebedev, Basic of ultrasonic nondestructive testing for stress measurement in solid material (Kiev, Naukova dumka. 1974)

5. GOST-R-52890-2007. Non-destructive testing. Acoustical method for stress testing in material of pipeline. General requeriments.

6. GOST-R-52731-2007. Non-destructive testing. Acoustical method for stress testing.

7. ASTM E 837-08. Standard Test Method for Determining Residual Stresses by the Hole-Drilling Strain-Gage Method.

8. Work instruction of Restan MTS3000 of SINT Technology srl., System for measuring residual stress by the hole-drilling method.

9. Y. Mirchev, P. Chukachev, M. Mihovski, Proceedings of the XVII National ScientificTechnical Conference “Acoustic 2015”, Sofia, Bulgaria 15,164 -166 (2015)

10. M. Mihovski, Y. Mirchev, P. Chukachev, V. Sergienko, AIP Conf. Proc. 1785, 040038 (2016) 